\title{
Buerger's Disease and Hyperhomocysteinemia: Is there a Relationship?
}

\author{
Pierpaolo Di Micco, ${ }^{*}$, Rosanna Di Fiore ${ }^{2,3}$, Gianluca Di Micco ${ }^{1}$, Giuseppe Cardillo ${ }^{2,3}$, Chiara Bellia ${ }^{4}$, \\ Sandro Quaranta ${ }^{2,3}$, Marcello Ciaccio ${ }^{4}$ and Giuseppe Castaldo ${ }^{2,3}$
}

\author{
${ }^{I}$ Buonconsiglio Fatebenefratelli Hospital of Naples, Naples, Italy \\ ${ }^{2}$ Dipartimento di Biochimica e Biotecnologie Mediche, Università di Napoli Federico II, Naples, Italy \\ ${ }^{3}$ CEINGE Biotecnologie Avanzate, Scarl, Naples, Italy \\ ${ }^{4}$ Cattedra di Biochimica Clinica, Università di Palermo, Italy
}

\begin{abstract}
Thromboangiitis obliterans, also known as Buerger's disease, is a cause of juvenile lower limb ischaemia. Buerger's disease is idiopathic and one of diagnostic criteria is the absence of atherosclerotic risk factors other than smoking. A possible involvement of thrombophilia has been investigated and the role of hyperhomocysteinemia is still matter of discussion. We describe 9 patients with Buerger's disease followed-up for the past 3 years. We found a significant increase in circulating homocysteine levels (mean: 31.6 in patients $v s 8.2 \mu \mathrm{mol} / \mathrm{L}$ in control subjects). We also analyzed the C677T mutation of MTHFR; 5/9 Buerger's patients were heterozygotes and 4/9 homozygotes for the mutation as compared with 3 heterozygotes in the control group. Our data, although preliminary, suggest a possible role of homocysteine in the pathogenesis of Buerger's disease as one of the causes of endothelial dysfunction. The role of MTHFR C677T variant must be further evaluated in larger trials involving patients with Buerger's disease.
\end{abstract}

Keywords: Thrombophilia, hyperhomocysteinemia, MTHFR, Buerger's disease.

\section{BACKGROUND}

Lower limb ischaemia is usually due to atherosclerosis. In a small percentage of cases non-atherosclerotic diseases may determine reduced blood flow to the lower limb and/or arterial occlusion [1]. Among the non-atherosclerotic diseases are thrombophilic disorders such as cardiac or aortic embolism, antiphospholipid syndrome, inflammatory chronic diseases, systemic sclerosis or acute or chronic vasculitis, in particular Buerger's disease. Buerger's disease is a thromboangiitis obliterans of arterial or venous vessels of medium size due to an idiopathic inflammatory disease [2]. No clinical symptom, nor laboratory or instrumental signs (e.g. radiological, ultrasound or angiographic imaging) are specific for Buerger's disease and a probability approach has been proposed [2]. Mozes et al. in 1970 proposed diagnostic criteria and scores including early onset (i.e. $<40$ years) and/or intermittent claudication and/or involvement of upper extremity vessels as the occurrence of migrating thrombophlebitis and/or Raynaud's phenomenon and/or radiological or histological features [3]. Similarly, Shionoya et al. suggested diagnostic criteria for Buerger's disease including early onset (i.e. < 40 years), history of smoking, intrapopliteal arterial occlusion, involvement of upper limb vessels, thrombophlebitis migrans and absence of further atherosclerotic risk factors other than smoking [4]. For this reason, patients with atherosclerotic risk factors other than smoking such as hypertension, dyslipidemia or diabetes usually did not show typical features of Buerger's disease. So, recently thombophilic

*Address correspondence to this author at the Internal Medicine and Emergency Room, Buonconsiglio Fatebenefratelli, Hospital of Naples, Naples, Italy; E-mail: pdimicco@libero.it conditions predisposing to a hypercoagulable state have been thought of as triggers for Buerger's disease [5].

The aim of this study was to search for a possible association between Buerger's disease and hyperhomocysteinemia in patients followed by our group.

\section{PATIENTS AND METHODS}

In the last 3 years, we selected 9 patients affected by Buerger's disease according to the diagnostic criteria described by Mozes and Shionoya [3, 4]. All patients underwent clinical observation for the onset of intermittent claudication with or without lower limb ischaemia. After examination by color-Doppler and angiographic evaluation all patients were assigned to treatment (pharmacological with or without surgical approaches).

We excluded patients with atherosclerotic risk factors other than smoking (i.e. age $>40$ years, hypertension, diabetes or dyslipidemia) and patients with a hypercoagulable state due to inherited and/or acquired thrombophilia (i.e. factor V Leiden gene variant, presence of A202120G prothrombin variant, acquired activated protein $\mathrm{C}$ resistance, antiphospholipid syndrome, protein $\mathrm{C}$ deficiency, protein $\mathrm{S}$ deficiency, antithrombin deficiency), cardiac arrhythmia or chronic autoimmune disease (e.g. systemic erythematosus lupus or systemic sclerosis).

All selected patients gave their written informed consent and the study was approved by a local Ethics Committee.

All patients were tested for fasting homocysteinemia and for MTHFR C677T genotype using "PCR real time" (MTHFR C677T LCSet and the Light Cycler 1.2 Instrument -Roche Diagnostics-). We studied 9 age- and sex-matched 
healthy subjects as a control group without a history for recent vascular disorders (e.g. acute coronary syndrome and/or venous thromboembolism in the last year). No participants were on vitamin supplementation or antithrombotic drugs.

Serum creatinine levels were recorded to exclude an acquired cause of hyperhomocysteinemia. Creatinine values were within the reference range in patients and control subjects $[1.05 \pm 0.20 \mathrm{mg} \backslash \mathrm{dL}($ mean $\pm \mathrm{SD})$ in patients $v s 0.95 \pm 0$. $22 \mathrm{mg} \backslash \mathrm{dL}$ in control subjects].

\section{STATISTICAL ANALYSIS}

All statistics tests were performed using MatLab 7.6.0 R2008a (The Matworks Inc., Natick, MA, USA). Homocysteine serum level is a continue variable: the Anderson-Darling test for normality and the Levene's test for homoscedasticity were used to assess the opportunity to use the Student's t-test. The Randomisation test and the Fligner-Policello test, a robust, modified Mann-Whitney-Wilcoxon rank-order test for populations, which assumes neither normality nor equal variances, were performed to test differences between patients and controls in serum homocysteine levels.

The Hardy-Weinberg Proportion was assessed in both groups. Test on genetic data was performed using a Fisher's exact test for $2 \times 3$ matrix, when genotype was considered, or for $2 \times 2$ matrix, when alleles were taken in to account. Finally, odds ratio (OR) computation with Bayesian credibility assessment were performed to test association between alleles and Buerger's disease.

\section{RESULTS}

Table 1 summarizes the homocysteine values and MTHFR C677T gene variant of the Buerger's patients.

The Buerger's patients had homocysteine mean levels of $31.6 \mu \mathrm{mol} / \mathrm{L}$ versus $8.2 \mu \mathrm{mol} / \mathrm{L}$ for the controls. The Anderson-Darling test showed that homocysteine levels were normally distributed in the controls but not in the patients (Table 2). Levene's test for variance equality showed heteroskedasticity (Table 2). So, the parametric Student's t-test was not applicable. The best Box-Cox transformation for patients group was logarithmic: using this normalization procedure the Anderson-Darling test showed a normal distribution in both groups, but Levene's test was still positive (data not shown). So, 2 distribution-free tests were used and both showed that mean and median homocysteine serum levels in patients and controls were significantly different (Table 2).

MTHFR C677T gene variant was present in all 9/9 patients compared with $3 \backslash 9$ of control subjects (Table 1). Homozygosity for MTHFR C677T gene variant was present in 4/9 patients compared to none of control subjects; heterozygosity was present in 5/9 patients compared with $3 \backslash 9$ of control subjects. Both groups were in Hardy-Weinberg Proportion but in opposite direction caused by the different frequency of the T allele. Table 3 shows that genotypes and alleles distribution between patients and controls groups were significantly different. The odds ratio computed for the $\mathrm{T}$ allele is significant OR is 13 and the $95 \%$ confidence interval does not encompass 1 and credible (the OR is greater than critical value computed by Bayesian credibility assessment). Finally, the $\Phi$ parameter of association is 0.5031 , showing that the $\mathrm{T}$ allele is a moderate risk factor.

\section{DISCUSSION}

Buerger's disease is a non-atherosclerotic occlusive disease characterised by thrombosis, inducing limb ischaemia and gangrene [2]. The disease is idiopathic and different from other immune arteritis, but there is a strong association with tobacco abuse [5]. On the other hand, hyperhomocysteinemia is a risk factor for both arterial and venous thrombosis, and also due to genetic defects such as MTHFR C677T gene variant and/or folate deficiency [6-9]. Tobacco abuse is also an important determinant of low folate levels [10]. In the past years, several association between Buerger's disease and alteration of haemostasis have been suggested [5]. In these reports a role of hyperhomocysteinemia playing a pathogenetic role in Buerger's disease was ruled out, but univocal data are lacking since the majority of reports were based on single case observations or small populations $[5,11,12]$. On the other hand, reports on the role of MTHFR C677T gene variant, a cause of hyperhomocysteinemia, as a trigger factor of Buerger's disease did not confirm this association $[13,14]$.

Table 1. Homocysteinemia and MTHFR C677T Gene Variant in the Patients with Buerger's Disease (Control Values are also Shown)

\begin{tabular}{|c|c|c|c|c|c|}
\hline Patients & Homocysteine $(\mu \mathrm{mol} / \mathrm{L})$ & MTHFR C677T Variant & Control Subjects & Homocysteine $(\mu \mathrm{mol} / \mathrm{L})$ & MTHFR C677T Varian \\
\hline M; age 29 years & 65 & Homozygous & $\mathrm{M}$; age 30 years & 9 & Wild type \\
\hline $\mathrm{M}$ age 25 years & 19 & Heterozygous & M; age 24 years & 6.8 & Wild type \\
\hline M; age 31 years & 34 & Heterozygous & M; age 31 years & 6.2 & Wild type \\
\hline M; age 37 years & 15 & Heterozygous & M; age 30 years & 7.5 & Heterozygous \\
\hline M; age 38 years & 71 & Homozygous & M; age 42 years & 10.4 & Heterozygous \\
\hline M; age 38 years & 25 & Homozygous & M; age 39 years & 9.4 & Heterozygous \\
\hline F; age 36 years & 25 & Heterozygous & F; age 38 years & 8.3 & Wild type \\
\hline M; age 32 years & 16 & Heterozygous & $\mathrm{M}$; age 33 years & 7.9 & Wild type \\
\hline Mean & 31.7 & & Mean & 8.2 & \\
\hline SD & 21.5 & & SD & 1.3 & \\
\hline
\end{tabular}


Table 2. Statistical Tests Applied to Assess Differences Between Patients and Controls Groups Homocysteine Serum Levels

\begin{tabular}{|c|c|c|}
\hline \multirow{2}{*}{ Applied Test } & \multicolumn{2}{|c|}{ Homocysteine } \\
\cline { 2 - 3 } & $\begin{array}{c}\text { Patients } \\
\text { Group }\end{array}$ & $\begin{array}{c}\text { Controls } \\
\text { Group }\end{array}$ \\
\hline \hline $\begin{array}{c}\text { Anderson-Darling test for normality } \\
\text { (p-value) }\end{array}$ & 0.0095 & 0.9928 \\
\hline $\begin{array}{c}\text { Levene's test for homoscedasticity } \\
\text { (p-value) }\end{array}$ & \multicolumn{2}{|c|}{0.0015} \\
\hline $\begin{array}{c}\text { Randomisation test on means } \\
\text { (1-tailed p-value) }\end{array}$ & 0.0001 \\
\hline $\begin{array}{c}\text { Fligner-Policello test on medians } \\
\text { (1-tailed p-value) }\end{array}$ & 0.00002 \\
\hline
\end{tabular}

Table 3. Statistical Tests Performed to Assess Difference in Genotype and Alleles Distributions Between Patients and Controls Groups

\begin{tabular}{|c|c|c|}
\hline MTHFR C677T Genotype & $\begin{array}{l}\text { Patients } \\
\text { Group }\end{array}$ & $\begin{array}{c}\text { Controls } \\
\text { Group }\end{array}$ \\
\hline $\mathrm{CC}$ & 0 & 6 \\
\hline $\mathrm{CT}$ & 5 & 3 \\
\hline TT & 4 & 0 \\
\hline Fisher's exact test (2-tailed p-value) & \multicolumn{2}{|c|}{0.0047} \\
\hline MTHFR C677T Alleles & $\begin{array}{l}\text { Patients } \\
\text { Group }\end{array}$ & $\begin{array}{l}\text { Controls } \\
\text { Group }\end{array}$ \\
\hline $\mathrm{C}$ allele & 5 & 15 \\
\hline $\mathrm{T}$ allele & 13 & 3 \\
\hline Fisher's exact test (2-tailed p-value) & \multicolumn{2}{|c|}{0.0020} \\
\hline Power & \multicolumn{2}{|c|}{0.9555} \\
\hline $\begin{array}{l}\text { Odds Ratio (OR) for } \mathrm{T} \text { allele } \\
\text { with } 95 \% \text { confidence interval }\end{array}$ & \multicolumn{2}{|c|}{$2.5919<13.0000<65.2037$} \\
\hline $\begin{array}{l}\text { Bayesian Credibility Assessment: } \\
\text { Critical Odds Ratio (COR) }\end{array}$ & \multicolumn{2}{|c|}{$\begin{array}{c}3.6828 \\
(\mathrm{OR}>\mathrm{COR} \text {. Test is credible } \\
\text { at the } 95 \%)\end{array}$} \\
\hline$\Phi$ & \multicolumn{2}{|c|}{$\begin{array}{c}0.5031 \\
\text { Moderate positive } \\
\text { association (risk factor) }\end{array}$} \\
\hline
\end{tabular}

Our data showed hyperhomocysteinemia in Buerger's patients that was significant when compared with control subjects. We can speculate on a pathogenetic role of hyperhomocysteinemia in Buerger's disease but also a secondary increase in homocysteine levels because of the endothelial dysfunction already present in subjects with Buerger's dis- ease cannot be excluded. On the other hand, the relationship with the MTHFR C677T gene variant reported here differs from previous published work [13, 14]. Further data are needed in larger populations with a wider ethnic distribution.

We suggest that the significant increase in homocysteine levels may have clinical implications if patients with Buerger's disease can benefit from vitamin supplementation (e.g. folic acid). This may provide a potential therapeutic option for these patients that frequently have a poor prognosis.

\section{ACKNOWLEDGEMENTS}

We gratefully acknowledge grants to CEINGE from: Regione Campania (contract for molecular diagnostics); MiUR (Ministry of University and Research), PS 126.

\section{REFERENCES}

[1] Adar R, Papa MZ, Scheiderman J. Thromboangiitis obliterans: an old disease in need of a new look. Int $\mathrm{J}$ Cardiol 2000; 75(S1): S167-S170.

[2] Fiessinger JN. Juvenile arteritis revisited. Pathophysiol Haemost Thromb 2002; 32: 295-8.

[3] Mozes M, Cahansky G, Doitsch V, Adar R. The association of atherosclerosis and Buerge's disease: a clinical and radiological study. J Cardiovasc Surg 1970; 11: 52-9.

[4] Shionoya S. Buerger's disease: diagnosis and management. Cardiovasc Surg 1993; 1: 207-14.

[5] Di Micco P, Niglio A, Scudiero O, et al. A case of Buerger's disease associated with MTHFR $_{\mathrm{C} 677 \mathrm{~T}}$ mutation homozygosity: a possible therapeutic support. Nutr Metab Cardiovasc Dis 2004; 14: 225 6.

[6] D’Uva M, Di Micco P, Strina I, et al. Hyperhomocysteinemia in women affected by unexplained sterility or recurrent pregnancy loss from Southern Italy: a preliminary report. Thromb J 2007; 5: 10 .

[7] Harmon DL, Woodside JV, Jarnell JW, et al. The common 'thermolabile' variant of methylene tetrahydrofolate reductase is a major determinant of mild hyperhomocysteinaemia. QJM 1996; 89: 5717.

[8] Martinelli I. Risk factors in venous thromboembolism. Thromb Haemost 2001; 86: 395-403.

[9] Kang SS, Wong PW, Norusis M. Homocysteinemia due to folate deficiency. Metabolism 1987; 36: 458-62.

[10] Piyathilake CJ, Macaluso M, Hine RJ, Richards EW, Krumdiek CL. Local and systemic effects of cigarette smoking on folate and vitamin B12. Am J Clin Nutr 1994; 60: 559-66.

[11] Caramaschi P, Biasi D, Carletto A, et al. Three cases of Buerger's disease associated with hyperhomocysteinemia. Clin Exp Rheumatol 2000; 18: 264-5.

[12] Stammler F, Diehm C, Hsu E, Stockinger K, Amendt K. The prevalence of hyperhomocysteinemia in thromboangioitis obliterans. Does homocysteine play a role pathogenetically? Dtscg Med Wochenschr 1997; 122: 1062-3.

[13] Glueck CJ, Haque M, Winarska M, et al. Stromelysin-1 $5^{\circ} \backslash 6^{\circ}$ and eNOS T-786C polymorphisms, MTHFR C677T and A1298C mutations, and cigarette-cannabis smoking: a pilot, hypothesisgenerating study of gene-enviroment pathophysiological associations with Buerge's disease. Clin Appl Thromb Haemost 2006; 12: 427-39.

[14] Kawasaki T, Fujimura H, Kakinoki E, Uemichi A, Miyata T. Is there a role for genetic polymorphism of C677T methyleneetetrahydrofolate reductase (MTHFR) in Buerger's disease? Thromb Haemost 2000; 84: 736-7. 